\title{
Effect of mild medical hypothermia on in vitro growth of Plasmodium falciparum and the activity of anti-malarial drugs
}

\author{
Khalid Rehman' ${ }^{1}$ Ulrich Sauerzopf ${ }^{1}$, Luzia Veletzky' , Felix Lötsch', Mirjam Groger ${ }^{1,2}$
}

and Michael Ramharter ${ }^{1,2,3^{*}}$ [D

\begin{abstract}
Background: Cerebral malaria remains a medical emergency with high mortality. Hypo-perfusion due to obstructed blood vessels in the brain is thought to play a key role in the pathophysiology of cerebral malaria leading to neurological impairment, long-term neuro-cognitive sequelae and, potentially, death. Due to the rapid reversibility of vascular obstruction caused by sequestered Plasmodium falciparum, it is hypothesized that mild medical hypothermia - a standard intervention for other medical emergencies-may improve clinical outcome. This preclinical in vitro study was performed to assess the impact of mild hypothermia on parasite growth and the intrinsic activity of anti-malarials drugs.
\end{abstract}

Methods: Three laboratory-adapted clones and two clinical isolates were used for growth assays and standardized drug sensitivity assessments using the standard HRP2 assay. All assays were performed in parallel under normothermic $\left(37^{\circ} \mathrm{C}\right)$, mild hypothermic $\left(32^{\circ} \mathrm{C}\right)$, and hyperthermic $\left(41^{\circ} \mathrm{C}\right)$ conditions.

Results: Parasite growth was higher under standard temperature condition than under hypo- or hyperthermia (growth ratio 0.85; IQR 0.25-1.06 and 0.09; IQR 0.05-0.32, respectively). Chloroquine and mefloquine had comparable in vitro activity under mild hypothermic conditions (ratios for IC50 at $37^{\circ} \mathrm{C} / 32^{\circ} \mathrm{C}: 0.88 ; 95 \% \mathrm{Cl} 0.25-1.50$ and 0.86 ; $95 \% \mathrm{Cl} 0.36-1.36$, respectively) whereas dihydroartemisinin was more active under mild hypothermic conditions (ratio for IC50 at $37^{\circ} \mathrm{C} / 32^{\circ} \mathrm{C}: 0.27 ; 95 \% \mathrm{Cl} 0.19-0.27$ ). Hyperthermia led by itself to almost complete growth inhibition and precluded further testing of the activity of anti-malarial drugs.

Conclusion: This preclinical evaluation demonstrates that mild medical hypothermia inhibits in vitro growth of $P$. falciparum and enhances the pharmacodynamic activity of artemisinin derivatives. Based on these preclinical pharmacodynamic data, the further clinical development of mild medical hypothermia as adjunctive treatment to parenteral artesunate for cerebral malaria is warranted.

Keywords: Malaria, In vitro, HRP2, Hypothermia, Drug activity, P. falciparum

\section{Background}

Cerebral malaria is a medical emergency requiring the immediate parenteral administration of effective antimalarial treatment in addition to best available intensive care. Cerebral malaria leads to high mortality and neurological and cognitive long-term sequelae even in settings

\footnotetext{
${ }^{*}$ Correspondence: michael.ramharter@medizin.uni-tuebingen.de ${ }^{3}$ Institut für Tropenmedizin, Universität Tübingen, Tübingen, Germany Full list of author information is available at the end of the article
}

where optimal intensive care is available [1]. The underlying pathophysiological mechanism is thought to be facilitated by the adherence of erythrocytes infected with Plasmodium falciparum trophozoites to the endothelial cell wall of small cerebral blood vessels with consecutive hypo-perfusion of the brain. This hypo-perfusion, which may lead to progressively increased intracranial pressure, is, however, rapidly reversible by the administration of anti-malarial drugs [2]. Clearance of parasites from peripheral blood is usually achieved within $24 \mathrm{~h}$ in most 
African settings, where the burden of severe malaria is highest-constituting a relatively short period of time for vulnerability of brain tissue [3].

Mild medical hypothermia is an established adjunctive treatment initiated immediately after successful resuscitation from sudden cardiac arrest. It has been demonstrated to improve neurological outcome in cardiac arrest survivors due to its ability to increase the permissive period of hypoperfusion of the brain and to attenuate reperfusion injury $[4,5]$. A target temperature of $32{ }^{\circ} \mathrm{C}$ is recommended for cardiac arrest patients and mild medical hypothermia is currently evaluated for other indications including ischemic stroke $[4,6]$.

Based on the analogy of the reversibility of hypoperfusion of the brain in cardiac arrest and in cerebral malaria, it is hypothesized that mild medical hypothermia has the potential to improve neurological and cognitive outcomes of patients treated for cerebral malaria. However, before testing this hypothesis in human patients, the preclinical evaluation of the effect of hypothermia on the growth of $P$. falciparum and the potential impact on the activity of anti-malarial drugs require investigation. Although hypothermia has been previously shown to reduce growth of $P$. falciparum $[7,8]$, this has not been investigated in the target range of mild medical hypothermia. Importantly, the effect of mild medical hypothermia on the pharmacodynamics of anti-malarial drugs has not been investigated before. This is particularly important as hypothermia may-at least in theory-lead to reduced anti-malarial activity due to reduced metabolic activity of the parasite and may thus put patients participating in such clinical trials at potential risk [9]. The aim of this study was, therefore, to evaluate the impact of hypothermia on the in vitro growth of P. falciparum and on the in vitro activity of anti-malarial drugs.

\section{Methods}

Five $P$. falciparum strains were used for this in vitro study, including three clones with distinct anti-malarial resistance patterns (7G8, DD2, 3D7) and two laboratory-adapted isolates from returning travellers from West Africa. Clone 7G8 is resistant to chloroquine and pyrimethamine. Clone DD2 shows resistance to pyrimethamine, mefloquine and chloroquine. Clone 3D7 is drug sensitive [10]. The laboratory-adapted strains were previously shown to exhibit high-level drug resistance against chloroquine but are sensitive to mefloquine and artemisinin derivatives. Parasites were kept in continuous malaria culture until optimum growth was achieved prior to growth inhibition assays as described previously $[11,12]$.

Growth inhibition assays were performed using a modified HRP2-assay protocol [13, 14]. All growth assays were performed in parallel under identical conditions except for incubation temperature. Temperatures were set at 37,41 and $32{ }^{\circ} \mathrm{C}$ using calibrated incubators. Culture plates were pre-dosed with ascending concentrations of the anti-malarial drugs chloroquine diphosphate (Sigma C6628), mefloquine and dihydroartemisinin. Stock solutions $(1 \mathrm{mg} / \mathrm{ml})$ of drugs were prepared in ethanol and further diluted to the required final concentrations (chloroquine $3200 \mathrm{nmol} / \mathrm{l}$, mefloquine $160 \mathrm{nmol} / \mathrm{l}$ and dihydroartemisinin $16 \mathrm{nmol} / \mathrm{l}$ ). Two-fold serial dilutions were employed and drug-free controls were performed in parallel.

Two-hundred microliter parasite culture with a parasitaemia of $0.01 \%$ and haematocrit of $1.5 \%$ were transferred to each of the drug-coated wells. All assays were performed in triplicate and negative controls were immediately frozen at initiation of the incubation period.

Growth was assessed by detection of HRP2 concentrations by ELISA using commercially available monoclonal antibodies $(1 \mu \mathrm{g} / \mathrm{ml}$ of IgM antibody MPFM-55 and IgG MPFG-55P55A, Immunology Consultants Laboratory, Portland, OR, USA). Inhibitory concentrations (IC) were calculated using freely available software (IVART) [15]. To obtain relative growth rates, logistic growth curves were fitted to data points obtained by serial dilution of lysed cultures using a least square regression model. Logistic regression analysis was conducted using the " $R$ " statistical package [16]. No ethical clearance was required for this in vitro study following regulations of the Ethics Committee of the Medical University of Vienna.

\section{Results}

Laboratory-adapted clones $(\mathrm{n}=3)$ and clinical isolates $(\mathrm{n}=2)$ were cultured in vitro until optimal growth was achieved. All parasites were then subjected in parallel to growth and growth inhibition assays under normothermic $\left(37^{\circ} \mathrm{C}\right)$, hyperthermia $\left(41^{\circ} \mathrm{C}\right)$ and mild hypothermic $\left(32{ }^{\circ} \mathrm{C}\right)$ conditions. All strains showed adequate growth under normothermic conditions as assessed by the increase of HRP2 concentrations in parasite culture (median increase 3.9; IQR 3-7.8). Hypothermia was associated with a markedly reduced growth rate compared to normothermia (relative growth rate 0.85 ; IQR $0.25-1.06$ ). Hyperthermia led to the highest inhibition of parasite growth (0.09; IQR 0.05-0.32); see (Table 1).

The anti-parasitic activity of the anti-malarial drugs chloroquine, dihydroartemisinin and mefloquine was assessed under the three respective temperature conditions (Table 1). Chloroquine and mefloquine had comparable IC50 values at 32 and $37{ }^{\circ} \mathrm{C}$ (chloroquine: $113 \mathrm{nmol} / \mathrm{l} ; 95 \%$ CI $2.8-224 \mathrm{nmol} / \mathrm{l}$ vs $137 \mathrm{nmol} / \mathrm{l}$; $95 \%$ CI $84-191 \mathrm{nmol} / \mathrm{l}$ and mefloquine $15 \mathrm{nmol} / \mathrm{l} ; 95 \%$ CI 5-25 nmol/l and $16 \mathrm{nmol} / \mathrm{l} ; 95 \%$ CI 9-24 nmol/l, 
Table 1 In vitro growth of Plasmodium falciparum and inhibitory concentrations of dihydroartemisinin chloroquine, and mefloquine under normo-, hypo- and hyperthermic conditions

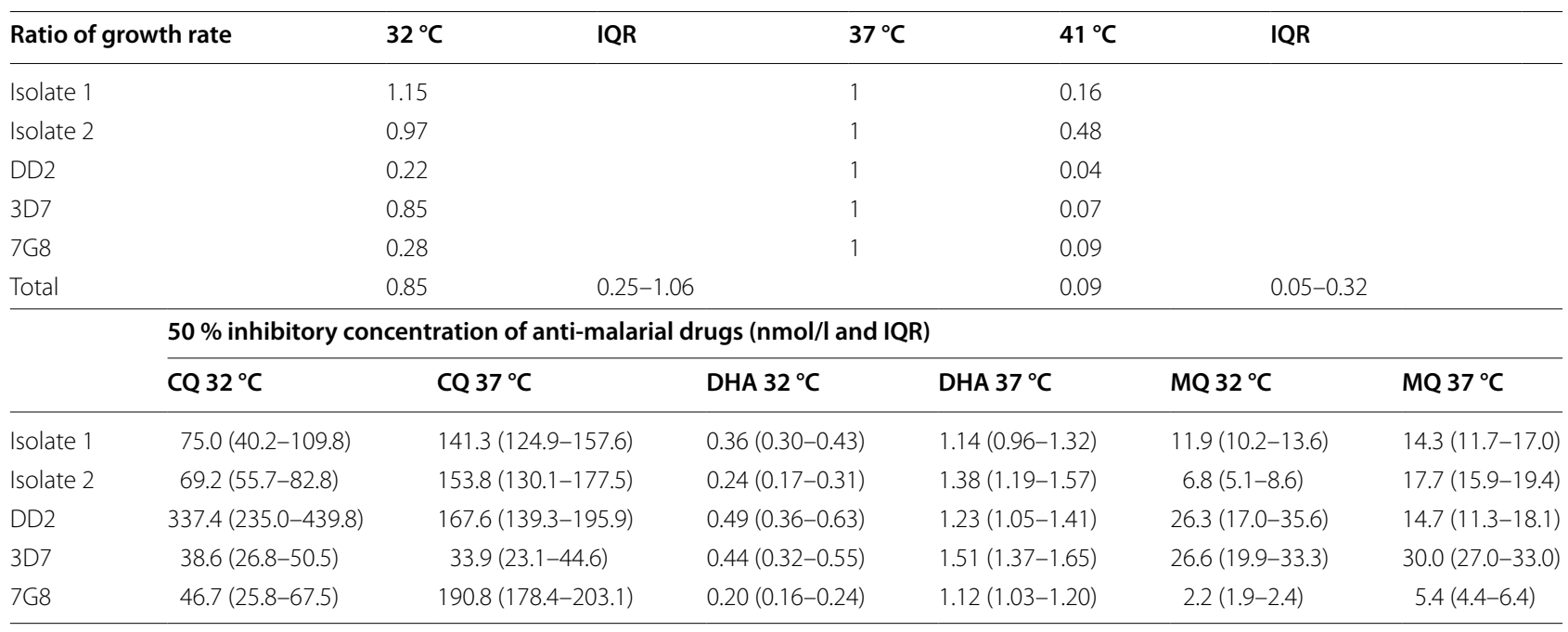

IQR interquartile range, $95 \% \mathrm{Cl} 95 \%$ confidence intervals, CQ chloroquine, $D H A$ dihydroartemisinin, $M Q$ mefloquine

respectively). However, dihydroartemisinin showed considerably lower IC50 values under mild hypothermic conditions $(0.3 \mathrm{nmol} / \mathrm{l} ; 95 \%$ CI $0.2-0.5 \mathrm{nmol} / \mathrm{l}$ vs 1.3 ; $95 \%$ CI 1.1-1.4 nmol/l; p < 0.01).

\section{Discussion}

This study demonstrates that mild hypothermia leads in itself to a considerable inhibition of parasite growth. This pharmacodynamic activity is independent of the action of anti-malarials and is therefore most likely unaffected by the presence of specific drug resistance. Interestingly, hyperthermia is associated with an even higher inhibition of in vitro parasite growth. However, hyperthermia is likely to increase the metabolic activity of brain tissue and therefore potentially leads to adverse outcome of cerebral malaria. Based on these data, the current clinical practice of normalizing temperature mechanically or with the use of antipyretic drugs may ultimately prove deleterious based on the finding of maximum in vitro parasite growth under normothermic temperature conditions. While the inhibitory effect of hyperthermia and hypothermia has been described previously, this is the first systematic evaluation of mild medical hypothermia and its inhibitory effect on P. falciparum in the context of anti-malarial drugs. These preclinical findings are further corroborated by clinical observations of faster parasite clearance in human patients in the absence of antipyretic drugs $[17,18]$.

It is well established that the early administration of parenteral artesunate is the most important treatment of severe malaria [1]. In vitro data from this study indicate that the anti-malarial activity of artesunate and its active metabolite dihydroartemisinin may be enhanced in two ways by mild hypothermia-firstly by direct inhibition of parasite growth and secondly by an increased anti-malarial activity of dihydroartemisinin when used under hypothermic conditions. Based on these findings there is-at least from a pharmacodynamic perspective-no convincing evidence precluding the use of mild medical hypothermia in the treatment of cerebral malaria patients. Whereas this study was performed with the concept of applying mild medical hypothermia in cerebral malaria, this pharmacodynamic activities may also apply to other forms of severe malaria. Similarly, the findings of retained or even increased anti-malarial activity as demonstrated for mefloquine, chloroquine and dihydroartemisinin in this in vitro study may apply to other classes of anti-malarials.

This study is a preclinical pilot study and is not designed to address all potential issues in the context of the use of medical hypothermia in malaria patients. Particularly the effect of mild medical hypothermia on cytoadherence can be investigated further in preclinical studies. Importantly, immunological consequences of hypothermia cannot be fully addressed by such in vitro experiments and the pharmacokinetics of anti-malarial drugs may potentially be altered by the use of mild medical hypothermia. These important aspects should therefore be diligently assessed in the context of the clinical development of hypothermia as adjunct treatment of cerebral malaria. Finally, the sample size of this pilot study is limited and the in vivo parasite reduction rate will therefore be an important outcome of future clinical trials [19]. 
The further development of mild therapeutic hypothermia may well continue directly in clinical trials in humans given the low predictability of animal models for cerebral malaria [20]. Whereas the conduction of such pilot studies in high-resource settings may be a viable option, endemic regions may be more relevant given the overall burden of disease. Importantly, external mechanical cooling of patients is feasible with relatively simple devices, including cooling blankets, but adequate monitoring of core temperature by oesophageal or urinary bladder probes is mandatory to avoid iatrogenic risks associated with hypothermia below $32{ }^{\circ} \mathrm{C}$ [4].

\section{Conclusion}

In summary, this in vitro study provides evidence supporting the clinical development of mild medical hypothermia for the treatment of cerebral malaria. Future studies should include feasibility outcomes of the procedure as well as pharmacodynamic and pharmacokinetic assessments associated with the use of mild medical hypothermia.

\section{Authors' contributions}

KR and MR conceived and designed the study. KR, US, LV, FL, and MG conducted the laboratory experiments. KR, US and MR performed the main data analysis. KR and MR drafted the manuscript. All authors read and approved the final manuscript.

\section{Author details}

1 Division of Infectious Diseases and Tropical Medicine, Department of Medicine I, Medical University of Vienna, Währinger Gürtel 18-20, 1090 Vienna, Austria. ${ }^{2}$ Centre de Recherches Médicales de Lambaréné, Hôpital Albert Schweitzer, Lambaréné, Gabon. ${ }^{3}$ Institut für Tropenmedizin, Universität Tübingen, Tübingen, Germany.

\section{Acknowledgements \\ We are grateful for the support of Heide-Maria Winkler. This study was sup- ported by the Karl Landsteiner Gesellschaft. We acknowledge the logistical support of the Global Infectious Disease Control Association. We acknowledge support by Deutsche Forschungsgemeinschft and Open Access Publishing fund of University Tuebingen.}

\section{Competing interests}

The authors declare that they have no competing interests.

Received: 26 December 2015 Accepted: 5 March 2016

Published online: 15 March 2016

\section{References}

1. Kurth F, Develoux M, Mechain M, Clerinx J, Antinori S, Gjørup IE, et al. Intravenous artesunate reduces parasite clearance time, duration of intensive care, and hospital treatment in patients with severe malaria in Europe: the TropNet severe malaria study. Clin Infect Dis. 2015:61:1441-4.

2. Seydel KB, Kampondeni SD, Valim C, Potchen MJ, Milner DA, Muwalo FW, et al. Brain swelling and death in children with cerebral malaria. N Engl J Med. 2015;372:1126-37.

3. WWARN Parasite Clearance Study Group. Baseline data of parasite clearance in patients with falciparum malaria treated with an artemisinin derivative: an individual patient data meta-analysis. Malar J. 2015:14(1):359.
4. Hypothermia after Cardiac Arrest Study Group. Mild therapeutic hypothermia to improve the neurologic outcome after cardiac arrest. N Engl J Med. 2002;346:549-56.

5. Zhang XW, Xie JF, Chen JX, Huang YZ, Guo FM, Yang Y, et al. The effect of mild induced hypothermia on outcomes of patients after cardiac arrest: a systematic review and meta-analysis of randomised controlled trials. Crit Care. 2015;19:417.

6. Wu T-C, Grotta JC. Hypothermia for acute ischaemic stroke. Lancet Neurol. 2013:12:275-84

7. Rojas MO, Wasserman M. Effect of low temperature on the in vitro growth of Plasmodium falciparum. J Eukaryot Microbiol. 1993:40:149-52.

8. Yuan L, Hao M, Wu L, Zhao Z, Rosenthal BM, Li X, et al. Refrigeration provides a simple means to synchronize in vitro cultures of Plasmodium falciparum. Exp Parasitol. 2014;140:18-23.

9. Wallmuller C, Herold B, Sterz F, Makristathis A, Ramharter M. Activity of antimicrobial drugs against bacterial pathogens under mild hypothermic conditions. Am J Emerg Med. 2015;33:1445-8.

10. Ramharter M, Noedl H, Thimasarn K, Wiedermann G, Wernsdorfer G, Wernsdorfer $\mathrm{WH}$. In vitro activity of tafenoquine alone and in combination with artemisinin against Plasmodium falciparum. Am J Trop Med Hyg. 2002;67:39-43.

11. Sauerzopf U, Honkpehedji YJ, Adgenika AA, Feugap EN, Ngoma GM, Mackanga JR, et al. In vitro growth of Plasmodium falciparum in neonatal blood. Malar J. 2014:13:436.

12. Ramharter M, Noedl H, Winkler H, Graninger W, Wernsdorfer WH, Kremsner PG, et al. In vitro activity and interaction of clindamycin combined with dihydroartemisinin against Plasmodium falciparum. Antimicrob Agents Chemother. 2003;47:3494-9.

13. Kurth F, Pongratz $P$, Bélard $S$, Mordmüller B, Kremsner PG, Ramharter $M$. In vitro activity of pyronaridine against Plasmodium falciparum and comparative evaluation of anti-malarial drug susceptibility assays. Malar J. 2009;8:1-6

14. Ramharter MNJ, Burkhardt D, Adegenika AA, Kremsner PG. In vitro activity of artemisone compared with artesunate against Plasmodium falciparum. Am J Trop Med Hyg. 2006;75:637-9.

15. Woodrow CJ, Dahlstrom S, Cooksey R, Flegg JA, Le Nagard H, Mentre F, et al. High-throughput analysis of antimalarial susceptibility data by the WorldWide Antimalarial Resistance Network (WWARN) in vitro analysis and reporting tool. Antimicrob Agents Chemother. 2013;57:3121-30.

16. Team RC. R: A language and environment for statistical computing. Vienna: R Foundation for Statistical Computing; 2014.

17. Lell B, Sovric M, Schmid D, Luckner D, Herbich K, Long HY, et al. Effect of antipyretic drugs in children with malaria. Clin Infect Dis. 2001;32:838-41.

18. Long HY, Lell B, Dietz K, Kremsner PG. Plasmodium falciparum: in vitro growth inhibition by febrile temperatures. Parasitol Res. 2001;87:553-5.

19. Angus B. Mild induced hypothermia for severe falciparum malaria. 2015. ISRCTN34508212. Clinicaltrials.gov. Accessed 1 December 2015.

20. White NJ, Turner GD, Medana IM, Dondorp AM, Day NP. The murine cerebral malaria phenomenon. Trends Parasitol. 2010;26:11-5.

Submit your next manuscript to BioMed Central and we will help you at every step:

- We accept pre-submission inquiries

- Our selector tool helps you to find the most relevant journal

- We provide round the clock customer support

- Convenient online submission

- Thorough peer review

- Inclusion in PubMed and all major indexing services

- Maximum visibility for your research

Submit your manuscript at www.biomedcentral.com/submit 